\title{
Perceptual overloading reveals illusory contour perception without awareness of the inducers
}

\author{
Marjan Persuh $^{1}$ - Tatiana Aloi Emmanouil ${ }^{2,3} \cdot$ Tony Ro $^{4}$
}

Published online: 1 June 2016

(C) The Psychonomic Society, Inc. 2016

\begin{abstract}
Unconscious perception is frequently examined by restricting visual input (e.g., using short stimulus durations followed by masking) to prevent that information from entering visual awareness. Failures to demonstrate perception without awareness may thus be a consequence of this restricted input rather than of limitations in unconscious perception. Here, we demonstrate a novel method that circumvents these significant drawbacks inherent in other methods. Using this new perceptual overloading technique (POT), in which stimuli are repeatedly presented in alternation with a stream of variable masks, we demonstrate illusory contour perception and modal completion even when subjects are completely unaware of the inducing elements. In addition to demonstrating a powerful new method to study consciousness by effectively gating robust visual input from visual awareness, we show that more complex contextual effects, previously considered to be a privilege only of conscious vision, can occur without awareness.
\end{abstract}

Keywords Illusory contours $\cdot$ Modal completion $\cdot$ Awareness

Marjan Persuh

mpersuh@bmcc.cuny.edu

1 Department of Social Sciences, Human Services and Criminal Justice, Borough of Manhattan Community College, City University of New York,

199 Chambers Street, New York, NY 10007, USA

2 Department of Psychology, Baruch College, City University of New York, B8215, Newman Vertical Campus, One Bernard Baruch Way, New York, NY 10010, USA

3 Program in Psychology, The Graduate Center of the City University of New York, New York, NY, USA

4 Programs in Psychology and Biology, The Graduate Center, City University of New York, 365 Fifth Ave, New York, NY 10016, USA
Understanding the depth and limits of unconscious processing represents one of the most challenging and important questions in psychology and neuroscience. In addition to illuminating the role of unconscious processing in human behavior, such knowledge might help us to better understand the role of consciousness in cognition. Although it is now widely accepted that low-level processing, such as the processing of color and shape (Breitmeyer, Ro, \& Singhal, 2004; Klotz \& Neumann, 1999; Ro et al., 2009; Schmidt, 2002), occurs unconsciously, evidence for unconscious high-level information processing remains mixed. For example, whereas some studies have shown cognitive control (e.g., conflict adaptation, error detection) without perceptual awareness (O'Connell et al., 2007; van Gaal, Lamme, \& Ridderinkhof, 2010), other studies suggest that awareness is necessary for these high-level processes (Ansorge, Fuchs, Khalid, \& Kunde, 2011; Kunde, 2003; Woodman, 2010). A possible reason for these discrepancies may be due to restricted visual inputs that are frequently used to study visual awareness rather than the necessity of awareness for higher level processing per se. In one illuminating example, when awareness was manipulated with visual masking (Breitmeyer, 1984), unconscious semantic processing occurred (Naccache \& Dehaene, 2001; Van den Bussche, Notebaert, \& Reynvoet, 2009); however, when awareness was manipulated with continuous flash suppression (CFS, Tsuchiya \& Koch, 2005), unconscious semantic processing was absent (Kang, Blake, \& Woodman, 2011).

In the current study, we tested whether modal completion occurs without perceptual awareness of the inducing context. Although it is well known that the perception of illusory shapes can emerge from context (Kanizsa, 1955), it is unclear whether such complex contextual effects occur in the absence of perceptual awareness, with a recent study suggesting that 
these effects do not emerge in the absence of awareness of the context (Harris, Schwarzkopf, Song, Bahrami, \& Rees, 2011). However, we reasoned that the lack of prior evidence for such effects might be due to methodological shortcomings rather than the necessity of visual awareness of the stimuli to produce these effects. Indeed, a major limitation for most studies measuring unconscious processing is that they use visual stimuli that are impoverished in many different ways. For example, studies often manipulate stimulus duration or the delay between the onset of a stimulus and the onset of a mask (Breitmeyer \& Öğmen, 2006), curtailing the amount of stimulus exposure time. Other studies, such as the one by Harris et al. (2011), use CFS, which presents a stimulus to one eye and a mask to the other, thereby limiting processing to early visual areas where monocular neurons are most abundant. Finally, studies using visual crowding (Bouma, 1970; Flom, Heath, \& Takahashi, 1963; Korte, 1923) present stimuli in the periphery amongst flankers, introducing large amounts of simultaneous noise in the visual input.

There are other well-known paradigms for rendering stimuli unconscious, such as inattentional blindness (Mack \& Rock, 1998) and motion-induced blindness (Bonneh, Cooperman, \& Sagi, 2001). These paradigms have been frequently employed to study the conditions and stimulus properties that restrict visual input from awareness. Although these approaches avoid stimulus degradation, they have other disadvantages that make them less desirable for studying unconscious processing. For example, studies on inattentional blindness typically require large numbers of participants because the critical stimulus is presented only on a single trial, whereas motion-induced blindness is difficult to manipulate because stimuli come in and out of awareness somewhat randomly. Consequently, these approaches have not been commonly used to study the limits of unconscious perception. Stimulus presentation limitations thus raise serious doubts regarding some of the conclusions that have been drawn from previous studies because it is likely that they have substantially underestimated the nature and extent of unconscious processing.

To overcome these limitations, we developed the perceptual overloading technique (POT), in which individual visual stimuli are presented in rapid alternation with noise masks for extended periods of time. In this paradigm, brief binocular presentations of stimuli, repeated over time and uncorrupted by simultaneous noise, allow information about these stimuli to accumulate, while the masks overload the visual system to prevent the critical stimuli from entering visual awareness. We hypothesized that if modal completion occurs in the absence of awareness of the context, this effect would be revealed with stimuli that are repeatedly presented yet not consciously perceived and would provide the first demonstration that illusory contours can be perceived without awareness of the inducers.

\section{Experiment 1}

In Experiment 1, we used POT to test whether modal completion occurs without conscious perception of the inducing elements. Current evidence (Harris et al., 2011) suggests that modal completion depends on the awareness of inducers, which is consistent with studies that demonstrate the essential role of feedback in modal completion (Gregory, 1972; Nakayama \& Shimojo, 1992; Wokke, Vandenbroucke, Scholte, \& Lamme, 2013).

\section{Method}

\section{Participants}

Twelve subjects (6 females), between the ages of 18 and 33 years $(M=21.7$ years $)$, were recruited from the undergraduate subject pool of the City College of the City University of New York and received course credit for their participation. All subjects reported normal or corrected-to-normal vision and participated in the experiment after giving informed consent. The sample size was determined based on a previous pilot study.

\section{Stimuli}

The stimuli were a fixation point, illusory shape inducers, and pattern masks (see Fig. 1) that were generated with the MATLAB Psychophysics Toolbox running on an Intel-based PC computer. The stimuli were presented at the center of a 17in. Sony Trinitron GS220 CRT monitor with the refresh rate set to $100 \mathrm{~Hz}$ and resolution set to $1,024 \times 768$. Monitor brightness was set to $40 / 100$ and contrast to $100 / 100$. The fixation point was a cross that subtended $0.38^{\circ}$, and the illusory shapes were squares and diamonds subtending $1.1^{\circ}$ that were formed by four inducers. Square and diamond illusory shapes appeared an equal number of times in the experiment. Inducers were bowtie shapes consisting of two sectors $\left(45^{\circ}\right)$ of a circle with a radius of $0.4^{\circ}$ that formed a right angle and a circle segment (radius $=0.7^{\circ}$ ). The circle segment had an arc length of $0.85^{\circ}$ and a height of $0.13^{\circ}$. The chord length matched the diameter $\left(0.8^{\circ}\right)$ of the circle sectors (see Fig. 1, inset). The pattern masks were composed of 10 pacman shapes (circles with radii of $0.4^{\circ}$ with $90^{\circ}$ sectors removed). The positions of the pacman shapes in the mask were random but with the constraint that eight pacmen partly overlapped with the eight possible inducer locations. Inducers were centered at the following locations: $\left(\left(-0.55^{\circ}, 0.55^{\circ}\right),\left(0^{\circ}, 0.78^{\circ}\right)\right.$, $\left(0.55^{\circ}, 0.55^{\circ}\right),\left(0.78^{\circ}, 0^{\circ}\right),\left(0.55^{\circ},-0.55^{\circ}\right),\left(0^{\circ},-0.78^{\circ}\right)$, $\left.\left(-0.55^{\circ},-0.55^{\circ}\right),\left(-0.78^{\circ}, 0^{\circ}\right)\right)$. The remaining two pacmen were randomly positioned within an imaginary square that was centered at fixation and subtended $2.1^{\circ}$. The orientation of each pacman was determined 


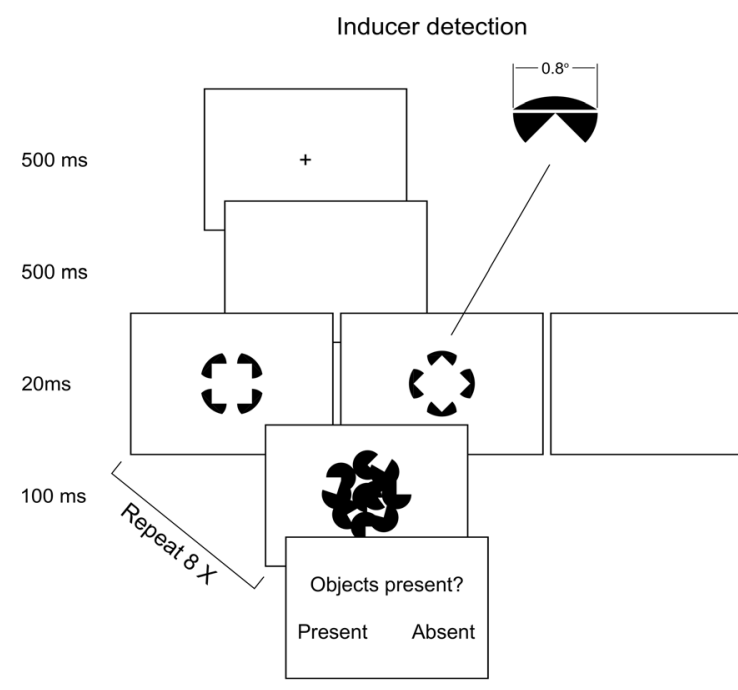

Fig. 1 Trial time course for the inducer detection (left) and shape discrimination (right) tasks in Experiment 1. Bowtie-shaped inducers (inset) or a blank screen were followed by a randomly generated pattern mask of

randomly. All stimuli were black $\left(\mathrm{L}=0 \mathrm{~cd} / \mathrm{m}^{2}\right)$ and were presented against a white background $\left(\mathrm{L}=85.3 \mathrm{~cd} / \mathrm{m}^{2}\right)$.

\section{Procedures}

The subjects were seated in a dimly lit, sound attenuated booth. A chin rest was used to prevent head movements and to maintain the distance between the eyes and monitor at $57 \mathrm{~cm}$. The experiment was divided into two halves that used the same stimuli. In the first half, participants were asked to detect the presence of the inducers, whereas in the second half participants discriminated between two illusory shapes (square and diamond). At the beginning of each trial, the fixation cross appeared for $500 \mathrm{~ms}$. Inducers (and, consequently, illusory shapes) were presented on only half of the trials for $20 \mathrm{~ms}$; blank screens of the same duration were presented on the other half of the trials. The inducers or blank screens were followed by a mask, which appeared for $100 \mathrm{~ms}$. The inducer-mask (or inducer-blank) cycle was repeated eight times, with a different pattern mask randomly generated for each cycle. Consequently, the positions of the inducers remained constant, but the positions of the pacman shapes in the mask varied across repetitions. Trials were separated by an intertrial interval of $500 \mathrm{~ms}$. The inducer-present and inducer-absent trials appeared in random order throughout the experiment. Participants first performed the inducerdetection task to avoid possible carryover effects in the form of clues regarding the presence of the inducers from perceptions of the illusory figures. The response prompt "Objects Present?" was presented with "Present, Absent" response options on the left and the right sides of the screen, in correspondence with the subject's response
Shape discrimination

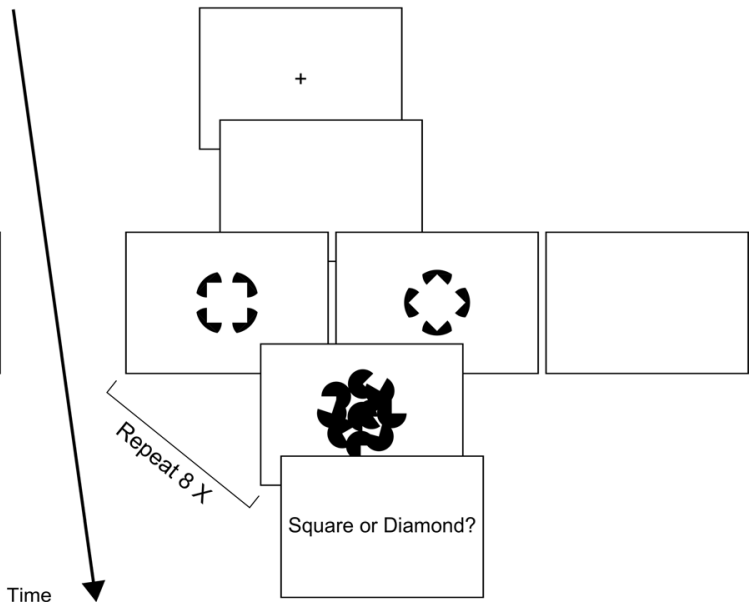

10 pacmen. Inducers (or a blank screen) and masks were presented in alternation eight times and were followed by a question and response alternatives, which remained on the screen until a response was made

button assignment. In the second part of the experiment, participants discriminated between a square and a diamond illusory shape. Participants entered their responses following the response prompt "Square or Diamond?," which was again in correspondence with their responsebutton assignment. Response assignments were counterbalanced across participants. For both tasks, participants entered their responses by pressing the 1 and 2 keys on the numeric keypad of the keyboard. Shape discrimination accuracy was calculated for inducer present trials only. For each part of the experiment, participants were first shown a demo, after which they completed one practice block of 12 trials and two experiment blocks of 30 trials. The demo for the inducer-detection task showed all possible inducers, presented sequentially, as well as the distractors. The demo for the shape-discrimination task consisted of six trials, three for each shape.

\section{Results}

To confirm that the inducers were effectively masked, we analyzed the data for the inducer-detection task, in which subjects reported the presence or absence of the inducers. Inducer-detection performance was no different than chance $(M=51.25 \%, S D=5.42 \%), t(11)=0.80, p=.441$, demonstrating the absence of awareness of the inducers (see Fig. 2). To rule out the possibility that chance performance in the inducer-detection task may have been due to biased reporting, we conducted a signal-detection analysis to compute a biasfree measure of perceptual sensitivity. A one-sample $t$ test confirmed that participants were at chance at detecting inducer presence, $d^{\prime}=0.08, t(11)=0.942, p=.367$, and that there was no response bias, $c=0.20, t(11)=1.524, p=.156$. 


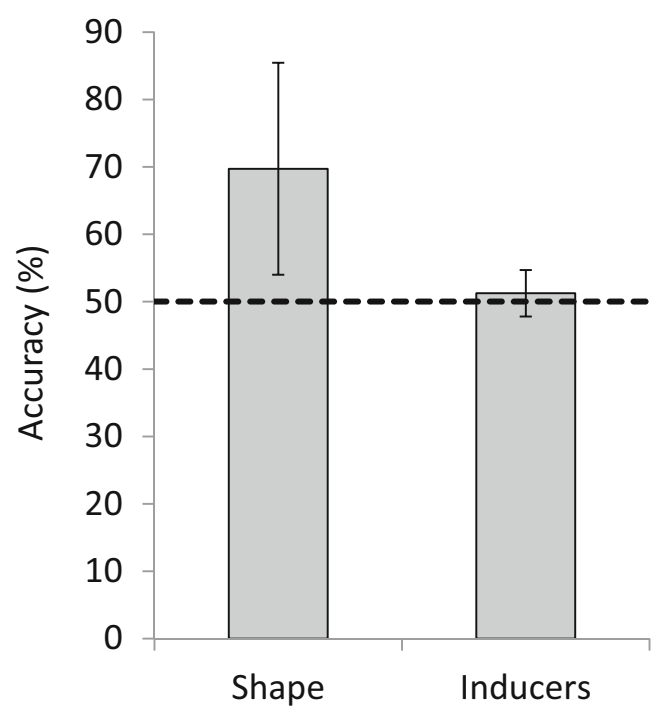

Fig. 2 Illusory shape discrimination and inducer-detection performance in Experiment 1. Illusory shape discrimination performance was above the $50 \%$ chance level (dashed line) on inducer-present trials. However inducer-detection performance was not significantly different from chance, demonstrating that inducers remained below the threshold for awareness. Error bars indicate 95\% confidence intervals

Although subjects were unaware of the presence of the inducers, analysis of the data from the shape-discrimination task revealed that illusory shape discrimination on inducerpresent trials was significantly above the $50 \%$ level expected by chance $(M=69.72 \%, S D=24.76 \%), t(11)=2.76, p=.019$, $d=0.8$. Signal-detection analysis confirmed that shape discrimination was above chance, $d^{\prime}=1.45, t(11)=2.933, p=$ .014 , and that responses were not biased, $c=0.06, t(11)=$ $0.393, p=.702$. These results indicate that participants perceived the illusory shapes even though masking prevented awareness of the inducers (see Fig. 2).

To further demonstrate that illusory shape perception can occur in the absence of awareness of the inducers, we assessed the relationship between inducer visibility and shape discrimination using a linear regression analysis (Greenwald, Draine, $\&$ Abrams, 1996). The absence of a correlation between inducer detectability and shape discriminability $(r=-.065, p=.841)$ is apparent in Fig. 3. The vertical intercept, which indicates shape discrimination at zero visibility, was significantly different from zero $\left(d^{\prime}=1.48, p=.020\right)$. These additional analyses demonstrate that illusory shape discrimination was independent from detectability of the inducers.

\section{Experiment 2}

To obtain additional evidence that the illusory shapes were perceived on the exact same trials that the inducers were not consciously processed, we conducted another experiment using a subjective measure of inducer awareness. In

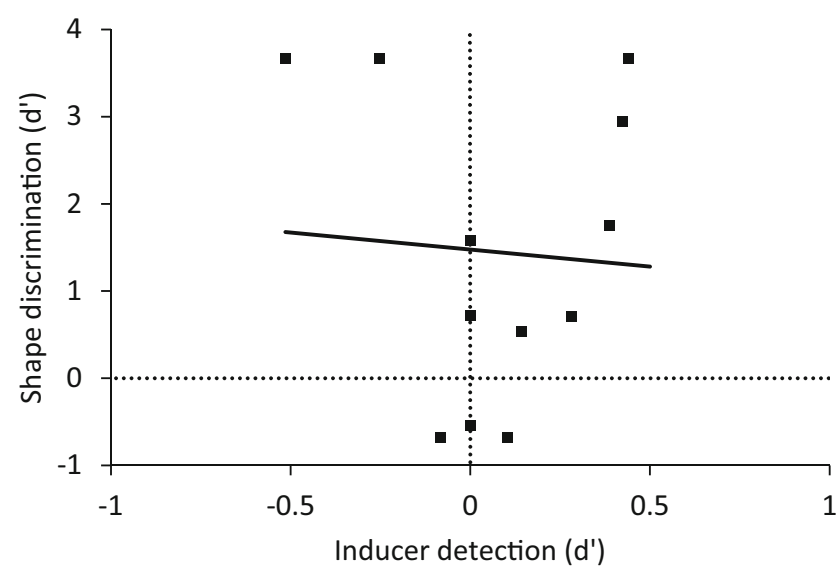

Fig. 3 Linear regression analysis of performance in the inducer-detection and shape-discrimination tasks in Experiment 1. There was no correlation between shape discrimination and inducer detectability, with significant shape discrimination at zero detectability (vertical intercept)

Experiment 2, participants reported the illusory shape and their awareness of inducers on each trial.

\section{Method}

\section{Participants}

Twelve subjects (eight females), between the ages of 20 and 31 years ( $M=24.2$ years), were recruited from the undergraduate subject pool of the City College of the City University of New York and received course credit for their participation. All subjects reported normal or corrected-to-normal vision and participated in the experiment after giving informed consent. One participant with a false alarm rate above $50 \%$ was excluded from analyses. The sample size was determined based on a previous pilot study.

\section{Stimuli and procedures}

The stimuli and procedure were identical to Experiment 1 except for the following. Inducers were present on $75 \%$ and absent on $25 \%$ of the trials. On each trial participants first reported illusory shape perception and then reported their level of awareness of the inducers (Overgaard, Rote, Mouridsen, \& Ramsøy, 2006; Sergent \& Dehaene, 2004). We used a perceptual awareness scale (PAS) in which subjects reported how they experienced the inducers using four ratings: $1=$ no experience, $2=$ brief glimpse, $3=$ almost clear experience, $4=$ absolutely clear experience. Our intention for using this scale was to examine responses at the extreme end of the scale (i.e., a rating of 1) to ensure that participants were not aware of the inducers. We did not focus on comparing performance for different ratings because we believe that the assumption that there are varying levels of perceptual awareness is controversial and incorrect (although the contents of awareness may 
vary in their perceptual qualities, awareness of a perceptual event itself does not vary). We therefore had subjects press 1 , 2,3 , or 4 on the keyboard to indicate their awareness rating and considered all responses that were not rated as 1 as being consciously perceived. A printed copy of PAS ratings was also available to participants for the duration of the experiment. Participants were first shown a demo, after which they completed one practice block of 16 trials and two experiment blocks of 32 trials.

\section{Results}

Not surprisingly, most subjects in Experiment 2 used almost exclusively a rating of 1 (no experience), which demonstrates that subjects were unaware of the inducers. On inducerpresent trials, a rating of 1 was used on $89.6 \%$ of the trials. On the remaining inducer-present trials, subjects used a rating of 2 on $8.3 \%$, a rating of 3 on $1.7 \%$, and a rating of 4 on $0.4 \%$ of the trials. On inducer-absent trials, subjects used a rating of 1 on $88.6 \%$ of the trials and reported a rating of 2 on $6.8 \%$, a rating of 3 on $4.0 \%$, and a rating of 4 on $0.6 \%$ of the trials.

We used only inducer-present trials on which subjects indicated no perceptual awareness (rating 1) to assess whether illusory shape perception can occur in the absence of awareness of the inducers (see Fig. 4). Consistent with Experiment 1, shape-discrimination performance was again significantly above chance $(M=64.03 \%, S D=16.44 \%), t(10)=2.83, p=$

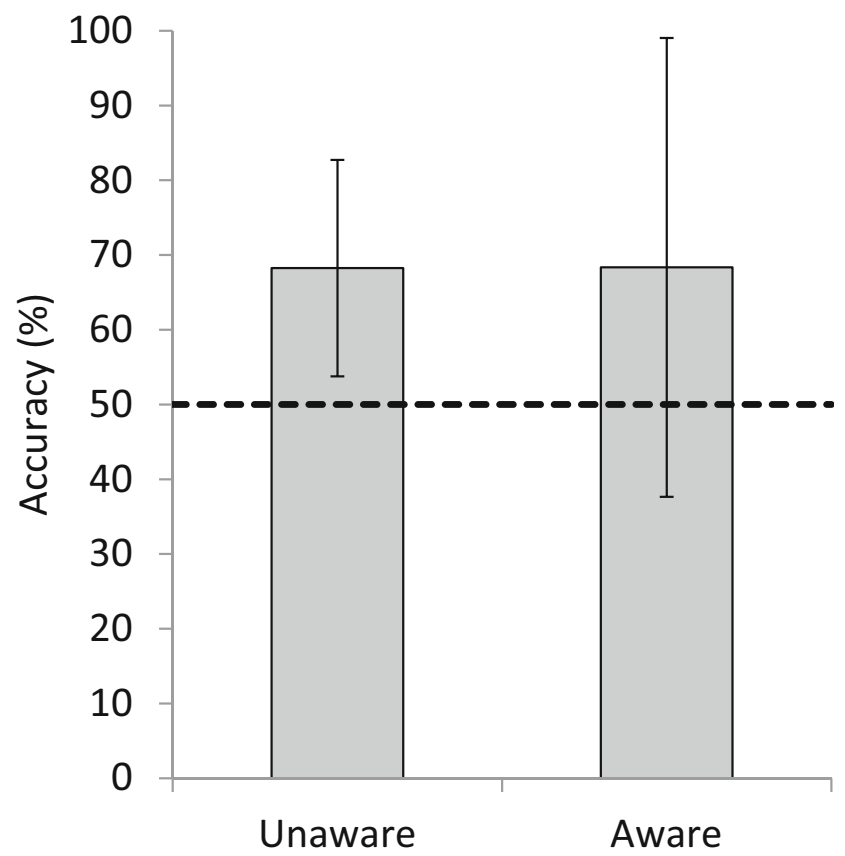

Fig. 4 Shape discrimination performance in Experiment 2. Illusory shape discrimination performance was above the $50 \%$ chance level on inducer-present trials. Note that the large variability in the aware conditions is due to the very small number of trials with these ratings. Error bars indicate $95 \%$ confidence interval
$.018, d=0.9$. Performance on trials with ratings of $2-4(M=$ $68.34 \%, S D=36.71 \%$ ) was almost identical to performance on trials with a rating of $1(M=68.24 \%, S D=17.32 \%)$ for the eight participants that used ratings of $2-4$ on at least some trials, $t(7)=0.006, p=.995$.

The results of Experiment 2 corroborate those of Experiment 1, showing that illusory shapes emerge from unperceived inducers masked with POT.

\section{Experiment 3}

We hypothesized that unconscious perception could be cumulative and that illusory shape perception without awareness of the inducers would only occur with multiple repetitions rather than with a single brief presentation, which is what is typically used in masking experiments to measure unconscious perception. To test this hypothesis, we compared illusory shape perception under varying numbers of inducer repetitions.

\section{Method}

\section{Participants}

Twelve subjects (eight females), between the ages of 18 and 22 years $(M=19.8$ years), were recruited from the undergraduate subject pool of the City College of the City University of New York and received course credit for their participation. All subjects reported normal or corrected-to-normal vision and participated in the experiment after giving informed consent. The sample size was determined based on a previous pilot study.

\section{Stimuli and procedures}

The stimuli and procedures were identical to Experiment 1 with the following modifications. Inducers were presented on every trial and were followed by a mask or a blank interval. The inducer-mask (or inducer-blank) sequence was presented 1, 2, 3,4 , or 8 times. The 10 conditions (mask/no mask $\times 1,2,3,4$, or 8 repetitions) were presented an equal number of times in random order across the experiment. At the beginning of the experiment, subjects received verbal instructions and were shown a 10 -trial demo version of the experiment. Next, subjects completed one practice block and then 10 experiment blocks, each comprised of 20 trials. In Experiments 1 and 2, we used two different measures of awareness and established that, with POT, participants were unaware of the inducers at the largest number of repetitions used in this experiment (i.e., eight). Because this experiment used eight or fewer repetitions, and because subjects were unaware of the inducers with eight cycles in the previous experiments, subjects should also not have conscious access to inducer information in this experiment. To confirm the lack of awareness of the inducers, we informed subjects after they 
completed the shape-discrimination task that the same inducers that were present on nonmasked trials were also present in the masked condition. They were then asked whether they saw, even slightly, these inducers in the masked trials. Their verbal responses were recorded by the experimenter.

\section{Results}

The accuracy data from Experiment 3 (see Fig. 5) were analyzed with a $2 \times 5$ ANOVA with masking condition (masked, nonmasked) and number of repetitions $(1,2,3,4,8)$ as the two within-subjects factors. There was a main effect of masking, $F(1,11)=55.41, p<.001, \eta_{\mathrm{p}}{ }^{2}=.834$, and of repetition number, $F(4,44)=4.63, p=.003, \eta_{\mathrm{p}}{ }^{2}=.296$. More importantly, there was a significant interaction between masking and number of repetitions, $F(4,44)=2.94, p=.031, \eta_{\mathrm{p}}{ }^{2}=.211$. In order to explain this interaction, we evaluated the effect of the number of repetitions separately for the masked and nonmasked conditions. The effect of repetition number was significant for the masked condition, $F(4,44)=3.91, p=.008$, $\eta_{\mathrm{p}}{ }^{2}=.262$, and there was a significant linear trend $F(1,11)=$ $9.54, p=.01, \eta_{\mathrm{p}}{ }^{2}=.464$. However there was no effect of repetitions in the nonmasked condition, $F(4,44)=1.79, p=$ .15. These results demonstrate that the perception of the illusory shapes emerged gradually with increasing number of repetitions in the masked condition but was consistently robust in the nonmasked condition (see Fig. 5).

To determine the number of repetitions that led to the perception of illusory shapes, we compared performance for each of the five repetitions in the masked condition with the $50 \%$

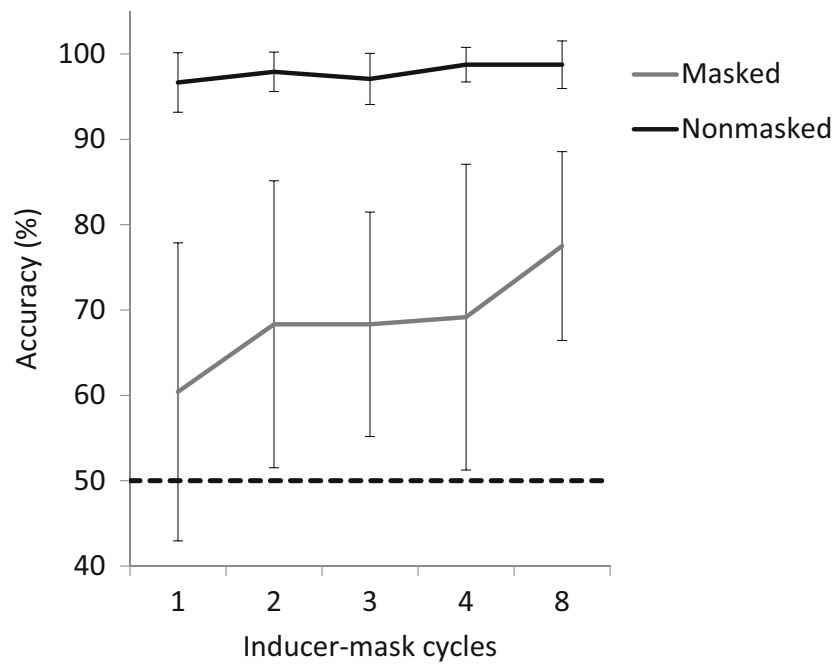

Fig. 5 Illusory shape discrimination for the masked and nonmasked conditions as a function of inducer mask repeats in Experiment 3. Illusory shape perception improved with increasing repeats in the masked but not the nonmasked condition. Performance was not significantly different from chance levels $(50 \%)$ with a single inducermask presentation in the masked condition. Error bars indicate $95 \%$ confidence intervals, corrected for multiple comparisons illusory shape discrimination level expected by chance. Paired-sample $t$ tests (Bonferroni corrected) showed that performance in the masked condition was significantly above chance with two or more repetitions (all $p \mathrm{~s}<.05$ ). Even when examining the data without the conservative Bonferroni correction, performance in the masked condition was not significantly above chance with only one cycle, $t(11)=1.85, p=$ .09 . Eleven of the 12 subjects $(92 \%)$ reported complete unawareness of inducers, and one subject reported that he might have seen inducers on some trials. The results were similar even when the data from the one subject who reported awareness of the inducers were excluded.

\section{Experiment 4}

We conducted a control experiment to exclude the possibility that participants were perceiving parts of the inducers rather than illusory contours. We compared discrimination of shapes created with inward-facing inducers, which produce strong illusory contours, with shapes composed of the same inducers rotated $180^{\circ}$, which produce minimal to no illusory contours (see Fig. 6). If performance depends on inducer visibility and not on contours, performance should be above chance with both types of inducers.

\section{Method}

\section{Subjects}

Twelve subjects (five females) between the ages of 18 and 31 years $(M=21.0$ years) participated in the experiment for course credit. All subjects were recruited from the undergraduate subject pool of the City College of New York, had normal or corrected-to-normal vision, and gave informed consent.

\section{Stimuli and procedure}

The stimuli and procedures were similar to those of Experiment 1 (see Fig. 6) with the following modifications. Each participant completed two blocks of shapediscrimination trials in which masked inducers were always present. In one block, the inducers were facing inwards, as in previous experiments; in a second block, the inducers were rotated by $180^{\circ}$, facing outwards. Outward-facing inducers produced very minimal to no illusory contours. Block order was counterbalanced between participants. In both blocks, participants were asked to discriminate between two possible shapes. After the target disappeared, the two possible shapes appeared on the left and right sides of the screen, with shape side counterbalanced across participants. Subjects pressed the 1 button on the keyboard to indicate that they thought the shape on the left side of the response screen was presented 
a

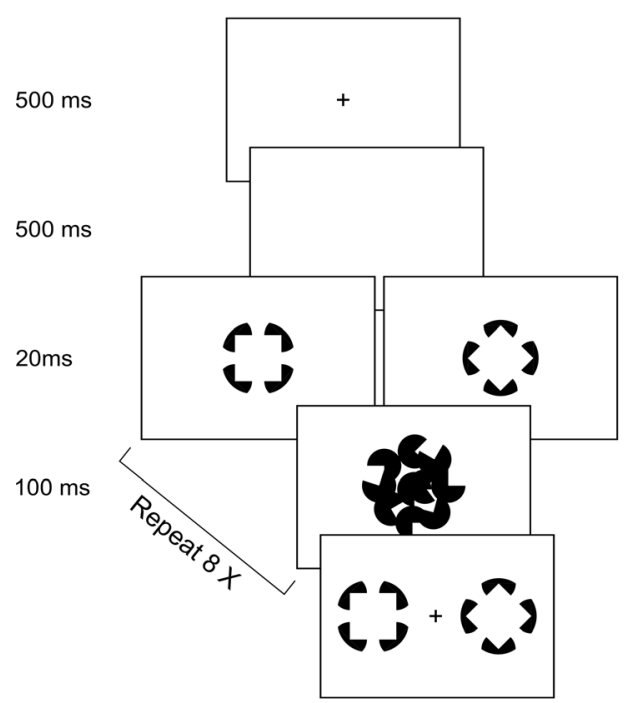

b

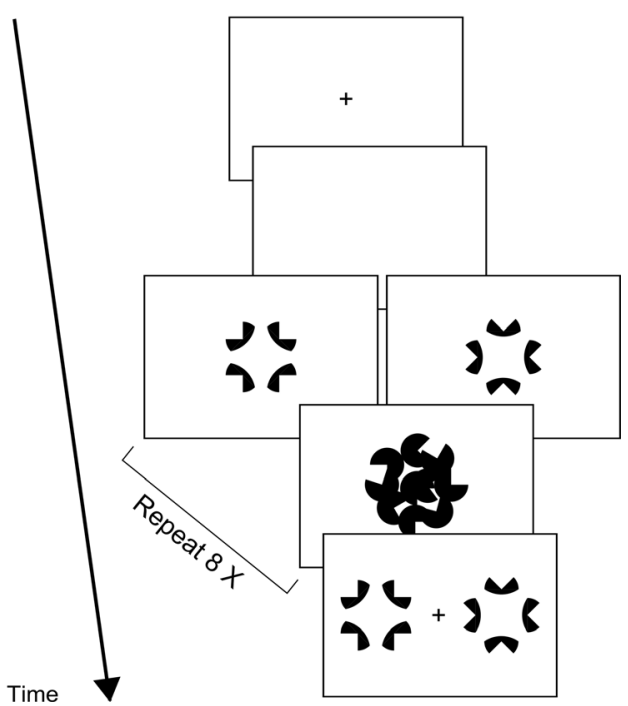

Fig. 6 Time course for trials in the shape-discrimination task for (A) inward-facing and (B) outward-facing inducers in Experiment 4. Response alternatives remained on the screen until the subjects made a selection

on that trial and the 2 button on the keyboard to indicate the shape on the right. The shapes remained on the screen until subjects made their response. To ensure that subjects were familiar with the shapes, they were shown a demo at the beginning of each block. Each shape was presented first by itself and then it was presented with a mask. We repeated this sequence three times for each shape so that subjects could experience the appearance of the shapes under masking conditions. Subjects then completed 12 practice trials and a block of 30 experimental trials.

\section{Results}

Shape-discrimination performance was significantly above chance for inward-facing inducers $(M=81.67 \%, S D=24.43$ $\%, t(11)=4.49, p=.001$, replicating the results of the first three experiments (see Fig. 7). However, performance on trials with outward-facing inducers was not significantly different from chance $(M=51.94 \%, S D=5.40 \%), t(11)=1.25, p=$ .239. Signal-detection analysis confirmed these results. For inward-facing inducers, shape-discrimination sensitivity was significant, $d^{\prime}=2.29, t(11)=4.54, p=.001$, with no response bias, $c=0.05, t(11)=0.971, p=.353$. For outward-facing inducers, sensitivity and response bias were not significantly different from zero, $d^{\prime}=0.10, t(11)=1.25, p=.239 ; c=0.08$, $t(11)=1.963, p=.075$. Some participants reported perception of faint contours and a slightly brighter stimulus area that together enabled them to perceive a square or diamond shape. Importantly, none of the participants perceived either luminance borders or a brighter stimulus area in the control condition with outward-facing inducers that did not produce illusory contours. Rather, participants reported perceiving only masking noise in these outward-facing inducer conditions. These results demonstrate that participants perceive illusory shapes under conditions with complete masking of the inducers.

\section{Discussion}

In this study we demonstrate perception of illusory contours and modal completion in the absence of awareness of the

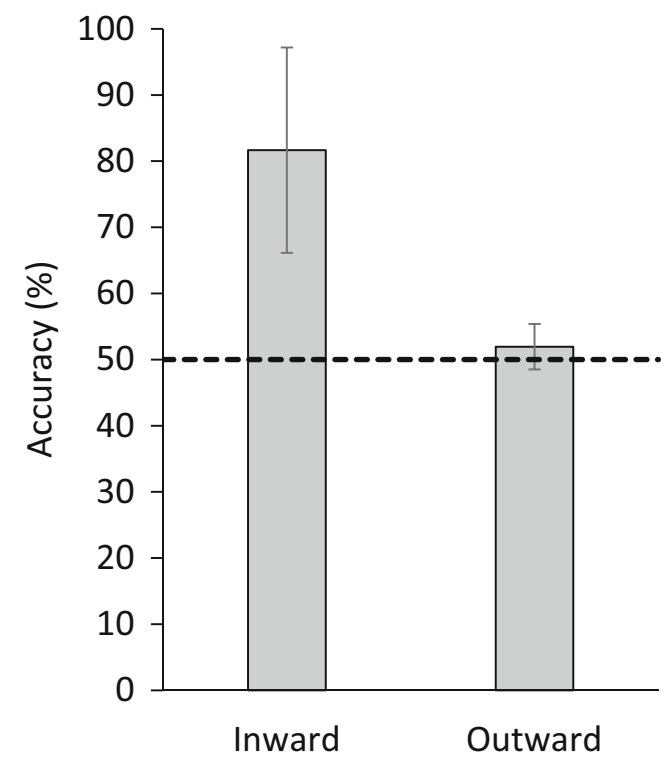

Fig. 7 Shape-discrimination performance for inward- and outwardfacing inducers in Experiment 4. Shape-discrimination performance was above the $50 \%$ chance level (dashed line) only for inward-facing inducers. Error bars indicate 95\% confidence intervals 
inducers. We hypothesized that the reason this and other complex perceptual processes have previously not been shown to occur without awareness may have been due to the limitations of current methods for studying unconscious vision (Kim \& Blake, 2005), which substantially degrade the visual input in order to block stimuli from awareness (but see Rahnev, Huang, \& Lau, 2012, for a paradigm that does not degrade the input of unattended stimuli). Using a new powerful method, the perceptual overloading technique (POT), we sought to overcome these limitations. In four experiments, we presented stimuli followed by pattern masks repeatedly, over an extended period of time. In all four experiments, we observed robust effects of the unconsciously perceived context on conscious perception of illusory contours. In Experiment 1 we used an objective measure of awareness and demonstrated that participants were unaware of the inducers despite perceiving illusory contours that were induced by them. In Experiment 2 we obtained converging evidence by employing a subjective measure of awareness. The results from these two experiments, which used different measures to assess awareness, similarly demonstrate that modal completion occurs without awareness of the inducing context. In Experiment 3 we extended these results and showed that perception of illusory shapes improves with repeated inducer-mask cycles. Experiment 4 further demonstrated the perception of these illusory contours without awareness of the inducers and ruled out the possibility that participants may have based their judgments on parts of inducers.

In contrast to our results, a recent study has claimed that modal completion requires awareness of the inducers (Harris et al., 2011). In that study, which employed continuous flash suppression (CFS), inducers were presented to one eye and were blocked from awareness by masks presented simultaneously to the other eye. Participants did not perceive any illusory shapes, suggesting that the masked inducers were not processed to a level that gives rise to illusory contours. However, it is likely that their negative result is due to several inherent limitations of the CFS stimuluspresentation method. First, because with CFS the inducers were presented to one eye along with noise to the other eye, information from the masks that was simultaneously presented with the inducers may have strongly interfered with illusory contour perception. Second, with CFS visual information processing is likely to be restricted to monocular neurons, which are most abundant in V1. Because neurophysiological studies show that computations underlying illusory contour formation occur in V2 and not V1 (von der Heydt, Peterhans, \& Baumgartner, 1984), the use of dichoptic presentations of inducers and masks that restrict inducer processing primarily to V1 neurons may have prevented illusory contour perception. In contrast to this previous
CFS study, our experiments presented the inducers and masks binocularly and in alternation, allowing for the unconscious processing of the inducers in binocular neurons and presumably in areas beyond $\mathrm{V} 1{ }^{1}$

Many other studies examining unconscious perception are likely to suffer from related methodological problems. For example, a number of experiments find dissociations between conscious and unconscious perception of features such as orientation (de Gardelle, Kouider, \& Sackur, 2010), color (Breitmeyer, Ro, Öğmen, \& Todd, 2007; Breitmeyer et al., 2004) and faces (de Gardelle, Charles, \& Kouider, 2011; de Gardelle \& Kouider, 2010). Although these dissociations may reflect differences between conscious and unconscious perception, it is also possible that they can be explained by the use of limited methods that provide brief or noisy visual input to prevent access to visual awareness. For example, with masking, stimulus presentation durations are greatly reduced, allowing very little time for stimulus processing. Other methods, such as CFS and crowding, allow for longer stimulus presentation times but include large amounts of additional concurrent stimuli noise to block certain stimuli from awareness. The perceptual overloading technique used in the current studies overcomes these limitations of other techniques by presenting stimuli binocularly and for longer durations (stimuli can be presented for several seconds), as well as in the absence of concurrent noise (stimuli and masks appear in alternation). We thus believe that POT may be instrumental in resolving the debates on high-level perceptual and cognitive processing without awareness and will allow for a better understanding of the limitations of unconscious perception.

Our study is consistent with evidence from a crowding study that showed that a lower luminance threshold is required to discriminate illusory shapes compared to individual inducer orientations (Lau \& Cheung, 2012), as well as with previous studies on neurologically impaired individuals that provide some evidence for modal completion in the absence of awareness of the inducers (Mattingley, Davis, \& Driver, 1997; Vuilleumier, Valenza, \& Landis, 2001). However, unlike our study, neither of these previous studies on neurologically impaired individuals completely eliminated awareness of all of the inducers and the evidence for modal completion was always indirect.

Other studies have shown rapid activation of motor responses by illusory contours that was independent of awareness (Seydell-Greenwald \& Schmidt, 2012) or have suggested that nonattended illusory contours may be perceptually experienced (Vandenbroucke, Sligte, Fahrenfort, Ambroziak, \& Lamme, 2012). Another study has provided some evidence

\footnotetext{
${ }^{1}$ We also demonstrated in a control experiment that POT is equally effective with binocular and monocular presentations with inducers presented to one eye followed by masks presented to the other eye, likely because the signal and noise are temporally segregated, creating a time window for the signal to be processed uncontaminated by noise.
} 
for perceptual grouping of illusory shapes without awareness (Wang, Weng, \& He, 2012). These studies show some effect of illusory contours in the absence of awareness; however, they do not contradict the claim made by Harris et al. (2011) that conscious modal completion requires awareness of the inducers. Our study is therefore the first to suggest that unconsciously presented inducers can be processed at a level deep enough to influence conscious perception as measured by direct report.

In conclusion, we were able to demonstrate conscious perception of illusory contours and modal completion in the absence of awareness of the inducing context using a powerful new approach, the perceptual overloading technique (POT). This method overcomes the drawbacks of previous methods and allows for more extensive processing of stimuli presented without awareness because it does not rely on single brief presentations or noisy signals. Using POT, we were able to show perceptual effects that were previously thought to require conscious perception of context. This new technique may reveal that other complex perceptual processes also occur in the absence of awareness when allowing for the sufficient processing of unconsciously presented stimuli.

Acknowledgments This research was supported by NSF grants 0843148 and 1358893 to Tony Ro.

Author contributions M. Persuh developed the study concept. All authors contributed to the study design. Data collection and analyses were performed by T. Emmanouil and M. Persuh. All authors participated in the writing of the manuscript.

\section{References}

Ansorge, U., Fuchs, I., Khalid, S., \& Kunde, W. (2011). No conflict control in the absence of awareness. Psychological Research, 75(5), 351-365. doi:10.1007/s00426-010-0313-4

Bonneh, Y. S., Cooperman, A., \& Sagi, D. (2001). Motion-induced blindness in normal observers. Nature, 411(6839), 798-801. doi:10.1038/ 35081073

Bouma, H. (1970). Interaction effects in parafoveal letter recognition. Nature, 226(5241), 177-178.

Breitmeyer, B. G. (1984). Visual masking: An integrative approach. New York, NY: Oxford University Press.

Breitmeyer, B. G., \& Öğmen, H. (2006). Visual masking. Oxford: Oxford University Press.

Breitmeyer, B. G., Ro, T., Öğmen, H., \& Todd, S. (2007). Unconscious, stimulus-dependent priming and conscious, percept-dependent priming with chromatic stimuli. Perception \& Psychophysics, 69(4), 550-557.

Breitmeyer, B. G., Ro, T., \& Singhal, N. S. (2004). Unconscious color priming occurs at stimulus- not percept-dependent levels of processing. Psychological Science: A Journal of the American Psychological Society/APS, 15(3), 198-202.

de Gardelle, V., Charles, L., \& Kouider, S. (2011). Perceptual awareness and categorical representation of faces: Evidence from masked priming. Consciousness and Cognition, 20(4), 1272-1281. doi:10.1016/ j.concog.2011.02.001 de Gardelle, V., \& Kouider, S. (2010). How spatial frequencies and visual awareness interact during face processing. Psychological Science, 21(1), 58-66. doi:10.1177/0956797609354064

de Gardelle, V., Kouider, S., \& Sackur, J. (2010). An oblique illusion modulated by visibility: Non-monotonic sensory integration in orientation processing. Journal of Vision, 10(10), 6-6.

Flom, M. C., Heath, G. G., \& Takahashi, E. (1963). Contour interaction and visual resolution: Contralateral effects. Science (New York, N.Y.), 142(3594), 979-980.

Greenwald, A. G., Draine, S. C., \& Abrams, R. L. (1996). Three cognitive markers of unconscious semantic activation. Science (New York, N.Y.), 273(5282), 1699-1702.

Gregory, R. L. (1972). Cognitive contours. Nature, 238(5358), 51-52.

Harris, J. J., Schwarzkopf, D. S., Song, C., Bahrami, B., \& Rees, G. (2011). Contextual illusions reveal the limit of unconscious visual processing. Psychological Science, 22(3), 399-405. doi:10.1177/ 0956797611399293

Kang, M.-S., Blake, R., \& Woodman, G. F. (2011). Semantic analysis does not occur in the absence of awareness induced by interocular suppression. The Journal of Neuroscience: the Official Journal of the Society for Neuroscience, 31(38), 13535-13545. doi:10.1523/ jneurosci.1691-11.2011

Kanizsa, G. (1955). Margini Quasi-Percettivi in Campi con Stimolazione Omogenea [Quasi-perceptual margins in homogeneously stimulated fields]. Rivista di Psicologia, 49, 7-30.

Kim, C.-Y., \& Blake, R. (2005). Psychophysical magic: Rendering the visible 'invisible'. Trends in Cognitive Sciences, 9(8), 381-388.

Klotz, W., \& Neumann, O. (1999). Motor activation without conscious discrimination in metacontrast masking. Journal of Experimental Psychology: Human Perception and Performance, 25(4), 976-992.

Korte, W. (1923). Uber die Gestaltauffassung im indirekten Sehen [On the apprehension of Gestalt in indirect vision]. Zeitschrift für Psychologie, 93, 17-82.

Kunde, W. (2003). Sequential modulations of stimulus-response correspondence effects depend on awareness of response conflict. Psychonomic Bulletin \& Review, 10(1), 198-205.

Lau, J. S. F., \& Cheung, S.-H. (2012). Illusory contour formation survives crowding. Journal of Vision, 12(6), 15-15.

Mack, A., \& Rock, I. (1998). Inattentional blindness. Cambridge, MA: MIT Press.

Mattingley, J. B., Davis, G., \& Driver, J. (1997). Preattentive filling-in of visual surfaces in parietal extinction. Science, 275(5300), 671-674. doi:10.1126/science. 275.5300 .671

Naccache, L., \& Dehaene, S. (2001). Unconscious semantic priming extends to novel unseen stimuli. Cognition, 80(3), 215-229.

Nakayama, K., \& Shimojo, S. (1992). Experiencing and perceiving visual surfaces. Science (New York, N.Y.), 257(5075), 1357-1363.

O’Connell, R. G., Dockree, P. M., Bellgrove, M. A., Kelly, S. P., Hester, R., Garavan, H.,...Foxe, J. J. (2007). The role of cingulate cortex in the detection of errors with and without awareness: A high-density electrical mapping study. The European Journal of Neuroscience, 25(8), 2571-2579. doi: 10.1111/j.1460-9568.2007.05477.x

Overgaard, M., Rote, J., Mouridsen, K., \& Ramsøy, T. Z. (2006). Is conscious perception gradual or dichotomous? A comparison of report methodologies during a visual task. Consciousness and Cognition, 15(4), 700-708.

Rahnev, D. A., Huang, E., \& Lau, H. (2012). Subliminal stimuli in the near absence of attention influence top-down cognitive control. Attention, Perception, and Psychophysics, 74(3), 521-532. doi:10. 3758/s13414-011-0246-Z

Ro, T., Singhal, N. S., Breitmeyer, B. G., \& Garcia, J. O. (2009). Unconscious processing of color and form in metacontrast masking. Attention, Perception \& Psychophysics, 71(1), 95-103. doi:10. 3758/app.71.1.95 
Schmidt, T. (2002). The finger in flight: real-time motor control by visually masked color stimuli. Psychological Science: A Journal of the American Psychological Society / APS, 13(2), 112-118.

Sergent, C., \& Dehaene, S. (2004). Is consciousness a gradual phenomenon? Evidence for an all-or-none bifurcation during the attentional blink. Psychological Science, 15(11), 720-728. doi:10.1111/j.09567976.2004.00748.x

Seydell-Greenwald, A., \& Schmidt, T. (2012). Rapid activation of motor responses by illusory contours. Journal of Experimental Psychology: Human Perception and Performance, 38(5), 11681182. doi: $10.1037 / \mathrm{a} 0028767$

Tsuchiya, N., \& Koch, C. (2005). Continuous flash suppression reduces negative afterimages. Nature Neuroscience, 8(8), 1096-1101. doi: $10.1038 / \mathrm{nn} 1500$

Van den Bussche, E., Notebaert, K., \& Reynvoet, B. (2009). Masked primes can be genuinely semantically processed: A picture prime study. Experimental Psychology, 56(5), 295-300. doi:10.1027/ 1618-3169.56.5.295

van Gaal, S., Lamme, V. A. F., \& Ridderinkhof, K. R. (2010). Unconsciously triggered conflict adaptation. PLOS ONE, 5(7), e11508-e11508.

Vandenbroucke, A. R. E., Sligte, I. G., Fahrenfort, J. J., Ambroziak, K. B., \& Lamme, V. A. F. (2012). Non-attended representations are perceptual rather than unconscious in nature. PLOS ONE, 7(11), e50042. doi:10.1371/journal.pone.0050042

von der Heydt, R., Peterhans, E., \& Baumgartner, G. (1984). Illusory contours and cortical neuron responses. Science (New York, N.Y.), 224(4654), 1260-1262.

Vuilleumier, P., Valenza, N., \& Landis, T. (2001). Explicit and implicit perception of illusory contours in unilateral spatial neglect: Behavioural and anatomical correlates of preattentive grouping mechanisms. Neuropsychologia, 39(6), 597-610.

Wang, L., Weng, X., \& He, S. (2012). Perceptual grouping without awareness: Superiority of Kanizsa triangle in breaking interocular suppression. PLoS ONE, 7(6), e40106. doi:10.1371/journal.pone. 0040106

Wokke, M. E., Vandenbroucke, A. R. E., Scholte, H. S., \& Lamme, V. A. F. (2013). Confuse your illusion: Feedback to early visual cortex contributes to perceptual completion. Psychological Science, 24(1), 63-71. doi:10.1177/ 0956797612449175

Woodman, G. F. (2010). Masked targets trigger event-related potentials indexing shifts of attention but not error detection. Psychophysiology, 47(3), 410-414. doi:10.1111/j.1469-8986.2009. 00948.x 